\title{
Electron-phonon relaxation and excited electron distribution in gallium nitride
}

Cite as: J. Appl. Phys. 120, 085708 (2016); https://doi.org/10.1063/1.4961874

Submitted: 06 May 2016 . Accepted: 16 August 2016 . Published Online: 30 August 2016

V. P. Zhukov, (D) V. G. Tyuterev, E. V. Chulkov, and P. M. Echenique

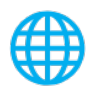

\section{ARTICLES YOU MAY BE INTERESTED IN}

Probing ultrafast carrier and phonon dynamics in semiconductors

Journal of Applied Physics 83, 1789 (1998); https://doi.org/10.1063/1.367411

Electron-phonon interaction and scattering in Si and Ge: Implications for phonon engineering Journal of Applied Physics 118, 045713 (2015); https://doi.org/10.1063/1.4927530

Luminescence properties of defects in GaN

Journal of Applied Physics 97, 061301 (2005); https://doi.org/10.1063/1.1868059

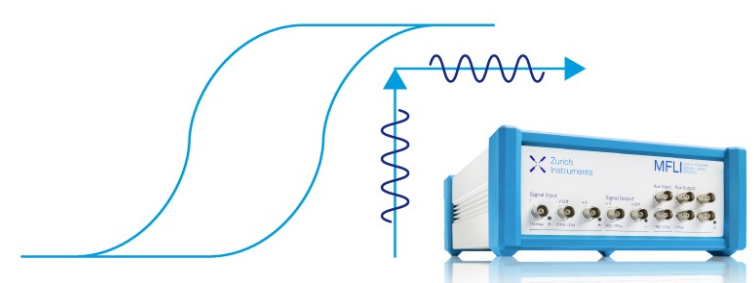

Webinar

How to Characterize Magnetic

Materials Using Lock-in Amplifiers

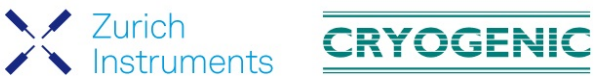




\title{
Electron-phonon relaxation and excited electron distribution in gallium nitride
}

\author{
V. P. Zhukov, ${ }^{1,2}$ V. G. Tyuterev, ${ }^{2,3,4, a)}$ E. V. Chulkov, ${ }^{2,4,5}$ and P. M. Echenique ${ }^{2,5}$ \\ ${ }^{1}$ Institute of Solid State Chemistry, Urals Branch of the Russian Academy of Sciences, Pervomayskaya st. 91 , \\ Yekaterinburg, Russia \\ ${ }^{2}$ Donostia International Physics Center (DIPC), P. Manuel de Lardizabal 4, 20018 San Sebastian, Spain \\ ${ }^{3}$ Tomsk State Pedagogical University, Kievskaya st. 60, Tomsk, Russia \\ ${ }^{4}$ Tomsk State University, Lenin st. 36, Tomsk, Russia \\ ${ }^{5}$ Departamento de Fisica de Materiales, Facultad de Ciencias Qumicas, UPVIEHU and Centro de Fisica de \\ Materiales CFM-MPC and Centro Mixto CSIC-UPVIEHU, Apdo. 1072, 20080 San Sebastian, Spain
}

(Received 6 May 2016; accepted 16 August 2016; published online 30 August 2016)

\begin{abstract}
We develop a theory of energy relaxation in semiconductors and insulators highly excited by the long-acting external irradiation. We derive the equation for the non-equilibrium distribution function of excited electrons. The solution for this function breaks up into the sum of two contributions. The low-energy contribution is concentrated in a narrow range near the bottom of the conduction band. It has the typical form of a Fermi distribution with an effective temperature and chemical potential. The effective temperature and chemical potential in this low-energy term are determined by the intensity of carriers' generation, the speed of electron-phonon relaxation, rates of inter-band recombination, and electron capture on the defects. In addition, there is a substantial high-energy correction. This high-energy "tail" largely covers the conduction band. The shape of the highenergy "tail" strongly depends on the rate of electron-phonon relaxation but does not depend on the rates of recombination and trapping. We apply the theory to the calculation of a non-equilibrium distribution of electrons in an irradiated GaN. Probabilities of optical excitations from the valence to conduction band and electron-phonon coupling probabilities in GaN were calculated by the density functional perturbation theory. Our calculation of both parts of distribution function in gallium nitride shows that when the speed of the electron-phonon scattering is comparable with the rate of recombination and trapping then the contribution of the non-Fermi "tail" is comparable with that of the low-energy Fermi-like component. So the high-energy contribution can essentially affect the charge transport in the irradiated and highly doped semiconductors. Published by AIP Publishing. [http://dx.doi.org/10.1063/1.4961874]
\end{abstract}

\section{INTRODUCTION}

The study of the relaxation processes in crystals is indispensable for the understanding of a number of physical phenomena induced by external sources at high excitation levels. First of all, this concerns to the experiments with the impact by electron beams and powerful lasers. ${ }^{1,2}$ The knowledge of the energy and momentum relaxation is also indispensable for the study of the high-field transport of charge carriers, ${ }^{3-5}$ including the case of electric breakdown. ${ }^{6,7}$ One should also mention such phenomena as laser ablation, ${ }^{8}$ abrupt thermal impact, ${ }^{9,10}$ ultra-fast phase transitions, ${ }^{11}$ photocatalysis, ${ }^{12}$ and the solar energy conversion. ${ }^{13,14}$ In the investigation of the energy relaxation processes in $\mathrm{Si},{ }^{1,15}$ $G a A s,{ }^{14,16-20} G a P,{ }^{21,22} \operatorname{InP},{ }^{21,23}$ and $C d S e,{ }^{24}$ the nonequilibrium distribution of electrons is usually simulated as a quasi-Fermi function which is localized close to the bottom of the conduction band.

In the works of Refs. 12 and 25-27 on the example of the compounds $\mathrm{ZnO}$ and $\mathrm{TiO}_{2}$, it was established that in a wide-bandgap material the evolution of energy relaxation

\footnotetext{
a) Author to whom correspondence should be addressed. Electronic mail: valtyut00@mail.ru
}

has some peculiarities. It has been shown that the form of quasi-equilibrium distribution differs markedly from the case of narrow-bandgap compounds, and covers a wide range of energy within the conduction and valence bands over the band edges at a distance comparable to the width of the band gap.

In the current article, we will consider the quasiequilibrium distribution in more general terms that allow us to incorporate into the theory ${ }^{27}$ the processes of radiative recombination and trapping of carriers. This makes it possible to specify the energy dependence of the distribution function either directly near the band edges or away from them. We apply the proposed theory to the case of gallium nitride in the wurtzite structure.

The paper is organized as follows. In Sec. II, we present the analytical methods we use. First, we derive the equation for the quasi-equilibrium distribution function. Next, its formal solution is given in Subsection II A. On this basis, in Subsection II B, we develop an effective phonon model that allows an exact solution which is presented in Subsection II C. Section III describes the techniques of numerical calculation for $G a N$. The necessary material dependent quantities, namely, optical excitation probabilities and electron-phonon coupling characteristics, are calculated $a b$ initio within the 
pseudopotential approach and density functional theory (DFT). Section IV presents the application of Secs. II and III to the non-equilibrium distribution of carriers in the conducting band of GaN. Finally, the results are discussed and summarized in Sec. V.

\section{CALCULATION METHODS}

The kinetic equation for the time-dependent distribution function $f(t, E)=G(E) n(t, E)$ of a non-equilibrium electron state created by the powerful external source of high-energy irradiation was investigated in Ref. 27. Here, $G(E)=$ $\sum_{c \mathbf{k}} \delta\left(E-E_{c \mathbf{k}}\right)$ is the density of electron states in the conduction band, $n(t, E)$ is the occupation number of nonequilibrium electrons, the energy $E$ is taken with respect to the bottom of the conduction band (hereafter "excess energy"). The excitation of electrons from the valence to conduction band by an external source is followed by the fast momentum randomization, within several femtoseconds. ${ }^{1}$ This conclusion was confirmed recently in the study of ultrafast scattering dynamics of hot electrons in GaAs by using time- and angle-resolved photoemission spectroscopy and $a b$ initio calculations. ${ }^{28}$ That is why, the occupation number $n_{c \mathbf{k}}$ of electron's band state $E_{c \mathbf{k}}$ can be regarded just as a function of energy $n(t, E)$, the phonon occupation number is considered to be energy-dependent as well: $N_{\sigma \mathbf{q}}=N(\epsilon)$.

We restrict ourselves to the case when the maximum excess energy of external excitation is located lower than the threshold of impact ionization $E_{i m p}$. Numerically, it roughly equals to the band-gap value above the bottom of the conduction band. Once the energy of an electron is lower than $E_{\text {imp }}$, the production of secondary electrons and holes is prohibited since it is impossible to simultaneously satisfy the energy and momentum conservation laws. Therefore, inelastic electron-electron processes are absent in this range, and energy relaxation can only be associated with the electronphonon scattering. For $G a N$, this threshold corresponds to the excess energy equal to $3.25 \mathrm{eV}$.

Retaining linear and quadratic terms by phonon energy which is a small quantity relative to the band energy $E_{c \mathbf{k}}$, one comes to the following equation for a non-equilibrium distribution $f(t, E)$ of electrons in the conduction band: ${ }^{27}$

$$
\begin{aligned}
\frac{d f(t, E)}{d t}= & {\left[\frac{d f(t, E)}{d t}\right]_{e x t}-G(E) n(t, E) \gamma_{r e c}(E) } \\
& +\frac{d}{d E}\left[\frac{d n(t, E)}{d E} \Gamma_{2}(E)+n(t, E)(1-n(t, E)) \Gamma_{1}(E)\right] .
\end{aligned}
$$

Hereafter,

$$
\begin{aligned}
& \Gamma_{0}(E)=\int_{0}^{\epsilon_{m}} \Gamma(\epsilon, E) d \epsilon ; \quad \Gamma_{1}(E)=\int_{0}^{\epsilon_{m}} \epsilon \Gamma(\epsilon, E) d \epsilon \\
& \Gamma_{2}(E)=\int_{0}^{\epsilon_{m}} \epsilon^{2}(N(\epsilon)+1 / 2) \Gamma(\epsilon, E) d \epsilon .
\end{aligned}
$$

A spectral function of electron-phonon interaction $\Gamma(\epsilon, E)$ is defined as

$$
\begin{aligned}
\Gamma(\epsilon, E)= & \sum_{\sigma \mathbf{q}} \sum_{c \mathbf{k} c^{\prime} \mathbf{k}^{\prime}} \delta\left(E-E_{c \mathbf{k}}\right) \times P_{c \mathbf{k} c^{\prime} \mathbf{k}^{\prime}}^{\sigma \mathbf{q}} \delta_{\mathbf{k}^{\prime} \pm \mathbf{q}-\mathbf{k}} \\
& \times \delta\left(E-E_{c^{\prime} \mathbf{k}^{\prime}}\right) \delta\left(\epsilon-\epsilon_{\sigma \mathbf{q}}\right) .
\end{aligned}
$$

$P_{c \mathbf{k} c^{\prime} \mathbf{k}^{\prime}}^{\sigma \mathbf{q}}=\frac{2 \pi}{\hbar}\left|\left\langle c \mathbf{k}\left|H_{\sigma \mathbf{q}}^{e l-p h}\right| c^{\prime} \mathbf{k}^{\prime}\right\rangle\right|^{2}$ is the matrix element of electron-phonon interaction operator. $\epsilon_{\sigma \mathbf{q}}$ is the energy of a phonon of $\sigma$ th branch with the wave vector $\mathbf{q}$. The term $[d f(t, E) / d t]_{\text {ext }}$ describes the distribution of excited carriers created by the external sources of generation. The expression $f(t, E) \gamma_{\text {rec }}(E)$ takes into consideration the flow of electrons from the conduction band to the valence bands and to the impurity levels. The range of integration in Eq. (2) extends from zero up to the maximal phonon energy $\epsilon_{m}$. The function,

$$
\gamma_{e-p h}(E)=\Gamma_{0}(E) / G(E),
$$

specifies the probability per unit time for an electron to leave a given excess energy level $E$, so it is the electron-phonon relaxation rate.

The well-known Eliashberg spectral function $\alpha^{2} F(\epsilon)$ of the electron-phonon interaction, which relates to the Fermilevel in superconductors, ${ }^{29}$ can be generalized for an arbitrary excess energy level in semiconductor as

$$
\alpha^{2} F(\epsilon, E)=\hbar \Gamma(\epsilon, E) /(2 \pi G(E)) .
$$

This function, in its turn, determines in semiconductor the electron-phonon coupling constant $\lambda(E)$, relating to a given energy $E$

$$
\lambda(E)=2 \int_{0}^{\epsilon_{m}} d \epsilon \alpha^{2} F(\epsilon, E) / \epsilon .
$$

\section{A. Stationary solution for the distribution function}

If the action of an external excitation continues for a time long enough, then the quasi-stationary distribution becomes established. Then a search of a quasi-stationary distribution function $d f(t, E) / d t=0$ reduces itself to the solution of the equation for the time-independent occupation number $n(E)$. The stationary solution of the nonlinear inhomogeneous differential equation (1) can be found by a perturbation theory. ${ }^{30}$ We seek the approximate stationary solution of Eq. (1) in the form $n(E)=n_{0}(E)+n_{1}(E)$. Here, $n_{0}(E)$ is the exact "non-perturbed" solution for a stationary homogeneous equation (1) with omitted sources and sinks,

$$
[d f(t, E) / d t]_{e x t}=0 ; \quad \gamma_{\text {rec }}(E)=0 .
$$

The first order correction $n_{1}(E)$ takes into account the terms $[d f(t, E) / d t]_{\text {ext }}$ and $\gamma_{\text {rec }}(E)$ treating them as a perturbation.

One can check by a direct substitution that there exists an exact partial solution for $n_{0}(E)$ in the following form:

$$
n_{0}(E)=\left(e^{R(E)}+1\right)^{-1},
$$

where 


$$
R(E)=\int_{0}^{E} \Gamma_{1}\left(E^{\prime}\right) / \Gamma_{2}\left(E^{\prime}\right) d E^{\prime}+C
$$

Here, $C$ is an integration constant.

Under the conditions of low intensity of external exposure, one has to regard $n_{0}(E)$ as a small quantity. In this case, keeping the leading terms we arrive from Eq. (1) to the linearized equation with a stationary solution,

$$
n_{0}(E)=e^{-R(E)} .
$$

Both distributions (8) and (10) should be localized in the energy region where the electron-phonon relaxation is inefficient, i.e., at $E \leq \epsilon_{m}$, where $\epsilon_{m}$ is the maximal energy of the phonon spectrum. An equation for the correction $n_{1}(E)$ comes out by substitution of $n_{0}(E)$ in Eq. (1). We restrict ourselves by the examination of excitation regimes when $n_{1}(E)$ can be treated as a small and smooth quantity. Retaining the leading terms, the equation for the correction term in the stationary regime acquires the following form:

$$
\begin{aligned}
& \frac{d}{d E}\left\{\left[1-2 n_{0}(E)\right] n_{1}(E) \Gamma_{1}(E)\right\}+[d f(t, E) / d t]_{e x t} \\
& \quad-n_{0}(E) G(E) \gamma_{\text {rec }}(E)=0 .
\end{aligned}
$$

The solution of Eq. (11) which satisfies the condition $n_{1}(E)=0$ at $E \geq E_{\max }$ can be written as

$$
\begin{aligned}
n_{1}(E)= & \left\{\left[1-2 n_{0}(E)\right] \Gamma_{1}(E)\right\}^{-1} \times \int_{E}^{E_{\max }}\left\{\left[d f\left(t, E^{\prime}\right) / d t\right]_{\text {ext }}\right. \\
& \left.-n_{0}\left(E^{\prime}\right) G\left(E^{\prime}\right) \gamma_{\text {rec }}\left(E^{\prime}\right)\right\} d E^{\prime} .
\end{aligned}
$$

Here, $E_{\max }$ is the maximal level of electrons excitation.

In a stationary regime, the total number of electrons generated per unit time by the external source should be equal to the number of electrons that leave the conduction band during the same time due to the inter-band recombination and trapping by the impurity levels

$$
\int_{0}^{E_{\max }}[d f(t, E) / d t]_{e x t} d E=\int_{0}^{E_{\max }} n_{0}(E) G(E) \gamma_{\text {rec }}(E) d E .
$$

Note that $\Gamma_{1}(E) \sim E$ as $E \rightarrow 0$. Hence, Eq. (13) provides the proper finite limit of $n_{1}(E)$ at the bottom of the conduction band. This equation defines implicitly the integration constant $C$ in Eq. (9)

As $n_{0}(E)$ is mostly localized in a small region of order of phonon spectrum width $E<\epsilon_{m}$ above the conduction band bottom then one can neglect the value $n_{0}(E) \ll 1$ at $E>\epsilon_{m}$.

The non-equilibrium occupation number $n_{1}(E)$ in the high energy region can be derived by using Eq. (13) which determines the integration constant $C$ in the solution (8) or (10) of $n_{0}(E)$,

$$
n_{1}(E) \approx \Gamma_{1}^{-1}(E) \int_{E}^{E_{\max }}\left[d f\left(t, E^{\prime}\right) / d t\right]_{e x t} d E^{\prime} .
$$

The expression similar to Eq. (14) for the high energy "tail" of the distribution function $f_{1}(E)=n_{1}(E) G(E)$ was formerly discussed in Refs. 12, 25, and 26, where the electron-hole recombination has not been formally taken into consideration. One can see now that the electron-hole recombination actually does not affect the shape of the high-energy "tail" of distribution.

\section{B. The "effective phonon" model}

Due to an extensive amount of complicated numerical calculations in Eqs. (2), (3), and (9), a straightforward derivation of the distribution function by Eqs. (8), (10), and (12) is prohibitively difficult. So we offer a simple model that adequately describes the scattering processes on the basis of some realistic averaging procedure.

Let us define an averaged energy loss per one electron in the process of a single transition act from an excess level $E$ as

$$
\epsilon_{a v}(E)=\Gamma_{1}(E) / \Gamma_{0}(E) .
$$

In calculations of Refs. 25 and 26, it was shown that for DFT band structures in $\mathrm{ZnO}$ and $\mathrm{TiO}_{2}$ this quantity manifests a weak energy dependence. In Sec. IV, we will show that this is also valid for $G a N$.

By using this observation, we introduce the model which neglects the energy dependence of $\epsilon_{a v}$ and considers that the electron interacts with a single averaged "effective" vibrational scatterer with a fixed energy $\epsilon_{0}$. In what follows, we identify $\epsilon_{0}$ with the numerically calculated $\epsilon_{a v}$. Next, we introduce the averaged electron-phonon coupling factor which we also calculate numerically by using the DFT band structure in Sec. IV,

$$
P_{a v}(E)=\Gamma_{0}(E) / G^{2}(E) .
$$

The formal averaging of (3) leads to the assumption,

$$
\Gamma(\epsilon, E)=P_{a v}(E) G^{2}(E) \delta\left(\epsilon-\epsilon_{0}\right) .
$$

Then the definition (4) implies that

$$
\gamma_{e-p h}(E)=G(E) P_{a v}(E) .
$$

Model expressions for $\Gamma_{0}, \Gamma_{1}, \Gamma_{2}$ follow from definitions (2)

$$
\begin{aligned}
& \Gamma_{0}(E)=P_{a v}(E) G^{2}(E) \\
& \Gamma_{1}(E)=\epsilon_{0} P_{a v}(E) G^{2}(E) \\
& \Gamma_{2}(E)=\epsilon_{0}^{2}\left(N\left(\epsilon_{0}\right)+1 / 2\right) P_{a v}(E) G^{2}(E) .
\end{aligned}
$$

Taking these approximations into account, we also find the expression for $R(E)$ as defined by Eq. (9)

$$
R(E)=E /\left[\epsilon_{0}\left(N\left(\epsilon_{0}\right)+1 / 2\right)\right]+C .
$$

Then Eq. (8) for $n_{0}$ acquires the form of quasi-Fermi distribution

$$
n_{0}(E)=\left(\exp \left(\frac{E-\mu}{k_{B} T_{e f f}}\right)+1\right)^{-1},
$$

where effective temperature of the excited electrons near the bottom of the conduction band is $T_{\text {eff }}=\epsilon_{0}\left(N\left(\epsilon_{0}\right)+1 / 2\right) / k_{B}$. 
The effective chemical potential $\mu=-k_{B} T_{e f f} C$ is defined implicitly by Equation (13).

\section{The case of weak degeneracy}

We discuss hereafter the case of week degeneracy, when $n_{0}(E) \ll 1$. This is a realistic case for the exposure by the sunlight or a mercury lamp, ${ }^{31}$ when the typical number of photons per unit cell of a crystal does not exceed $10^{-5}$. In this case, Eq. (10) reduces to the quasi-Boltzmann distribution

$$
n_{0}(E)=A \exp \left(-E /\left[\epsilon_{0}\left(N\left(\epsilon_{0}\right)+1 / 2\right)\right]\right) .
$$

For further simplification, let us introduce the separable approximation for the emission term $[d f(t, E) / d t]_{e x t}$ in Eq. (14),

$$
[d f(t, E) / d t]_{e x t}=S_{0}(t) \bar{S}(E) .
$$

Here, the function $S_{0}(t)$, in general time-dependent, is defined to be equal to the total number of excited electrons per unit of time, which depends on the intensity of exposure. In the quasi-stationary regime, $S_{0}(t)$ changes slowly, $d S_{0} / d t \ll S_{0}(t) / \tau_{e-p h}$, and can be considered as a constant $S_{0}(t) \approx S_{0}$.

The function $\bar{S}(E)$ describes the distribution of the excited electrons over the energy scale of the conduction band. It has to be normalized to unity: $\int \bar{S}(E) d E=1$. Employing this definition and effective phonon approximation, one can write the expression for the "tail" distribution as

$$
n_{1}(E) \approx \frac{S_{0}}{\epsilon_{0} \gamma_{e-p h}(E) G(E)} \int_{E}^{E_{\max }} \bar{S}\left(E^{\prime}\right) d E^{\prime} .
$$

The first-principle calculations demonstrate that in $G a N$ near the bottom of the conduction band $G(E)$ has almost linear energy dependence. We approximate this dependence as $G(E)=G_{0} E$.

In general, $N\left(\epsilon_{0}\right)$ is also a non-equilibrium distribution of phonons, and it would be necessary to investigate its evolution in conjunction with the evolution of the electron distribution. However, if the duration of action of external excitation exceeds the characteristic relaxation time of the phonon subsystem, it can be regarded as the Boltzmann function.

In the low-temperature limit when $N\left(\epsilon_{0}\right) \ll 1$, we can neglect a phonon occupation number. We also neglect the energy dependence of the recombination and trapping rate $\gamma_{\text {rec }}(E)$ in the small energy range $\epsilon_{m}$ near the bottom of the conduction band, hence $\gamma_{\text {rec }}(E)=\gamma_{\text {rec }}$. In the high excitation regime, the value $E_{\max } \gg \epsilon_{m}$ and in Eq. (13) it can be replaced by infinity. This helps us, employing Eq. (13), to find the normalization coefficient $A$ in expression (22) for $n_{0}$

$$
A=4 S_{0} /\left(\gamma_{\text {rec }} G_{0} \epsilon_{0}^{2}\right) .
$$

Now we see that the $n_{0}(E)$ depends on the intensity of exposure, via the function $S_{0}$, as well as on the rate $\gamma_{\text {rec }}$ of trapping and recombination processes.
Hence, the total electron distribution function,

$$
f(E)=f_{0}(E)+f_{1}(E)=G(E)\left(n_{0}(E)+n_{1}(E)\right),
$$

is written as

$$
\begin{aligned}
f(E)= & S_{0}\left[\left\{4 \gamma_{\text {rec }}^{-1} E / \epsilon_{0}-\gamma_{e-p h}^{-1}(E)\right\} \times \exp \left(-2 E / \epsilon_{0}\right)\right. \\
& \left.+\gamma_{e-p h}^{-1}(E) \int_{E}^{E_{\max }} \bar{S}(E) d E\right] / \epsilon_{0} .
\end{aligned}
$$

\section{TECHNICAL DETAILS}

We apply the described approach to the gallium nitride compound in the wurtzite structure. Our calculations have been performed by employing the $a b$ initio pseudo-potential computer code Quantum Espresso (QE) based on the density functional theory for the electron band structure and densityfunctional perturbation theory for the phonon band states. ${ }^{32}$ The pseudo-wave functions were expanded in plane waves with the energy cutoff of $820 \mathrm{eV}$. In the calculations of the electron states, we employed a set of 50 wave vectors per irreducible part of the Brillouin zone (IPBZ). The calculations of the phonon states and characteristics of the electron-phonon coupling were performed for a set of 12 wave vector in the IPBZ. Gallium norm-conserving atomic pseudo-potential was calculated by using the Bachelet-Hamann-Schlter method ${ }^{33}$ with Perdew-Zunger exchange-correlation potential. ${ }^{34}$ The pseudo-potential for nitrogen was constructed within the approach of Von Barth and Car. ${ }^{35}$ We will show later that such a way of calculations provide very good results for both electron and phonon band structures.

We compute the $\bar{S}(E)$ distribution function also based on the band structure calculations by means of the QE computer code. For example, if the energy of the quantum of optical excitation is $E_{\text {exc }}$ then for the excess energy $E$ we sum the probabilities of all direct excitations from the electronic states at the energy $E-E_{\text {exc }}$ to the states at the energy $E$. Hence, the non-normalized $\bar{S}(E)$ function is

$$
\bar{S}(E)=\sum_{\mathbf{k} n n^{\prime}} \delta\left(E-E_{n \mathbf{k}}\right) T\left(n \mathbf{k}, n^{\prime} \mathbf{k}\right) \delta\left(E-E_{e x c}-E_{n^{\prime} \mathbf{k}}\right),
$$

where $T\left(n \mathbf{k}, n^{\prime} \mathbf{k}\right)$ is the probability of the transition between the states $|n \mathbf{k}\rangle$ and $\left|n^{\prime} \mathbf{k}\right\rangle$. For the calculations of the $T\left(n \mathbf{k}, n^{\prime} \mathbf{k}\right)$ transition probabilities, we apply dipole approximation. The details of such an approach can be found in Ref. 36.

\section{RESULTS AND DISCUSSION}

The calculated dispersion curves and the corresponding total density of electron states are given for $\mathrm{GaN}$ in Fig. 1 . The direct band gap in $\Gamma$-point is equal to $3.25 \mathrm{eV}$, in good agreement with the experimental value of $3.4 \mathrm{eV}$ (Ref. 37). The top of the valence band is formed by $2 p$-like states of nitrogen, the bottom of the conduction band corresponds to the $4 s$-like states of $G a$. Also, the energy differences between the conduction band states at symmetry points of the Brillouin zone agree with the results of previous 


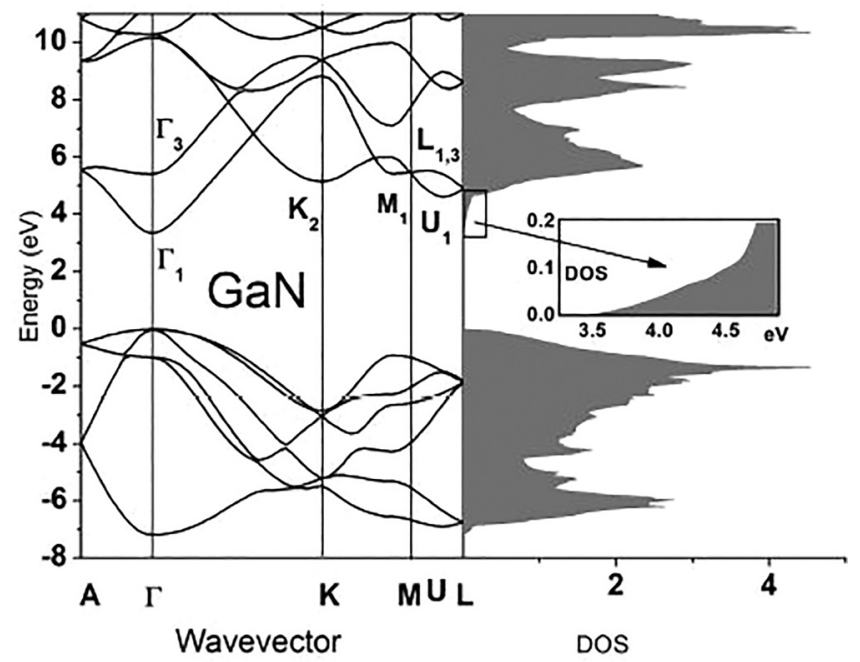

FIG. 1. Electron dispersion curves and density of states for GaN. The inset displays DOS at the bottom of a conduction band.

calculations. $^{38}$ For example, our calculated $\Gamma_{1}-K_{2}, \Gamma_{1}$ $-L_{1,3}, \Gamma_{1}-M_{1}$ differences are equal to $1.8,1.55,2.06 \mathrm{eV}$, whereas Bulutay et al. ${ }^{38}$ obtained 1.6, 1.75, $1.87 \mathrm{eV}$, respectively. The conduction band at $\Gamma_{1}$ displays the non-parabolic dispersion, so the density of states versus the excess electron energy deviates from the free-electron-like law $\sim_{\sqrt{E}}$ practically immediately above the bottom of the conduction band. The non-parabolicity of the conduction band states has been revealed experimentally in Ref. 39. Up to the excess energy of about $1.25 \mathrm{eV}$, which is up to the energy in the central $\Gamma_{1}$ valley lying between the side $L_{1,3}$ and $M_{1}$ states, the total DOS displays almost linear dependence (see for inset in Fig. 1 ), so near the bottom of the conduction band we approximate it with $G(E)=G_{0} E$ where $G_{0}=0.025 \mathrm{eV}^{-2}$.

In Fig. 2, our calculated phonon dispersion curves are compared with the experimental data of Ref. 40. Similar to the theoretical results of this paper, our computed frequencies are systematically about 5\% higher than the experimental ones. We demonstrate in this figure the calculated data scaled with the factor 0.95 . In Fig. 2, we also show the corresponding density of the phonon states. In the following

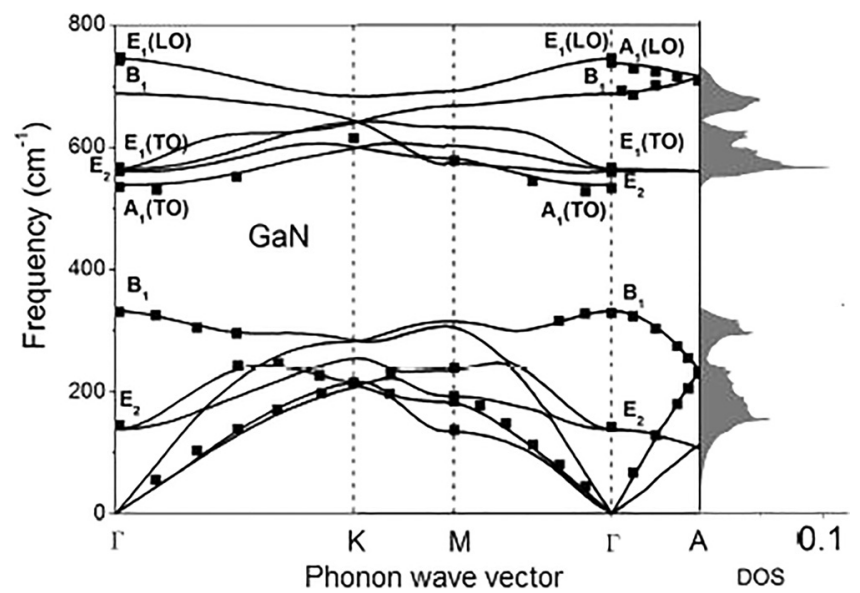

FIG. 2. Phonon dispersion curves and DOS for GaN. Experimental data are taken from Ref. 40. calculations, the highest energy of the phonon spectrum is taken to be equal to $785 \mathrm{~cm}^{-1}=0.097 \mathrm{eV}$.

Hereafter, we discuss the electron-phonon relaxation at excess energies which do not exceed the impact ionization level $3.25 \mathrm{eV}$ in $\mathrm{GaN}$. The inter-valley electron-phonon scattering events happen at higher energies and are not a subject of the current study.

In Fig. 3, we show the Eliashberg function calculated for three different values of excess energy in interval from 0.03 to $1.83 \mathrm{eV}$. We see that with increasing excess energy the electron-phonon scattering becomes stronger. For the states near the bottom of the conduction band, the scattering of excess electrons energy occurs mainly via emission of optical phonons, both transversal $(T O)$ and longitudinal $(L O)$ branches, being in correspondence with the common opinion on the main role of such phonons in the processes of electron energy relaxation. ${ }^{41} \mathrm{We}$ see, however, that with rising electron excess energy the contribution of acoustic branches essentially increases. In contrast to Ref. 42 where the dominating scattering via emission of the $L O$ phonons was supposed to occur, we find that at any value of the excess energy the contribution of the $T O$-branches is almost equal to that of the longitudinal ones.

In Fig. 4, we demonstrate the energy dependence of the $\lambda$, $\gamma_{e-p h}, \epsilon_{a v}$ values. The dependencies of $\lambda$ and $\gamma_{e-p h}$ also demonstrate the strengthening of electron-phonon scattering with the rise of the excess energy. However, the effective phonon energy $\epsilon_{a v}$ only slightly changes, so in the estimation of the relaxation rate and distribution curves we use the energyaverage value $\epsilon_{0}=0.085 \mathrm{eV}$. We see in the Eliashberg curves that the $\epsilon_{0}$ value lies just between the bands of the $L O$ and $T O$ branches which at any value of the excess energy have almost equal contributions to the electron relaxation rate.

According to Eq. (18), the change in the average lifetime of a single electron $\tau_{e-p h}(E)=\gamma_{e-p h}^{-1}(E)$ with energy is determined by the changes in the density of states $G(E)$ and in the averaged probability of phonon emission $P_{a v}(E)$. In Fig. 5, we show $\tau_{e-p h}(E), G(E)$, and $P_{a v}(E)$ versus the excess energy.

We find a very rapid change of $P_{a v}(E)$ at the energy less than $\sim 0.25 \mathrm{eV}$. But at a higher energy $P_{a v}(E)$ rather slowly decreases with energy while $G(E)$ rapidly increases that

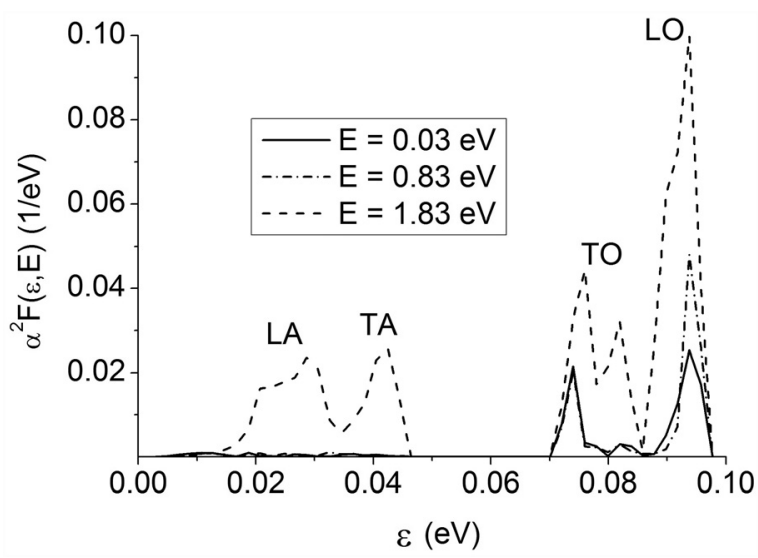

FIG. 3. Generalized Eliashberg function $\alpha^{2} F(\varepsilon, E)$ for three different values of the electron excess energy. 

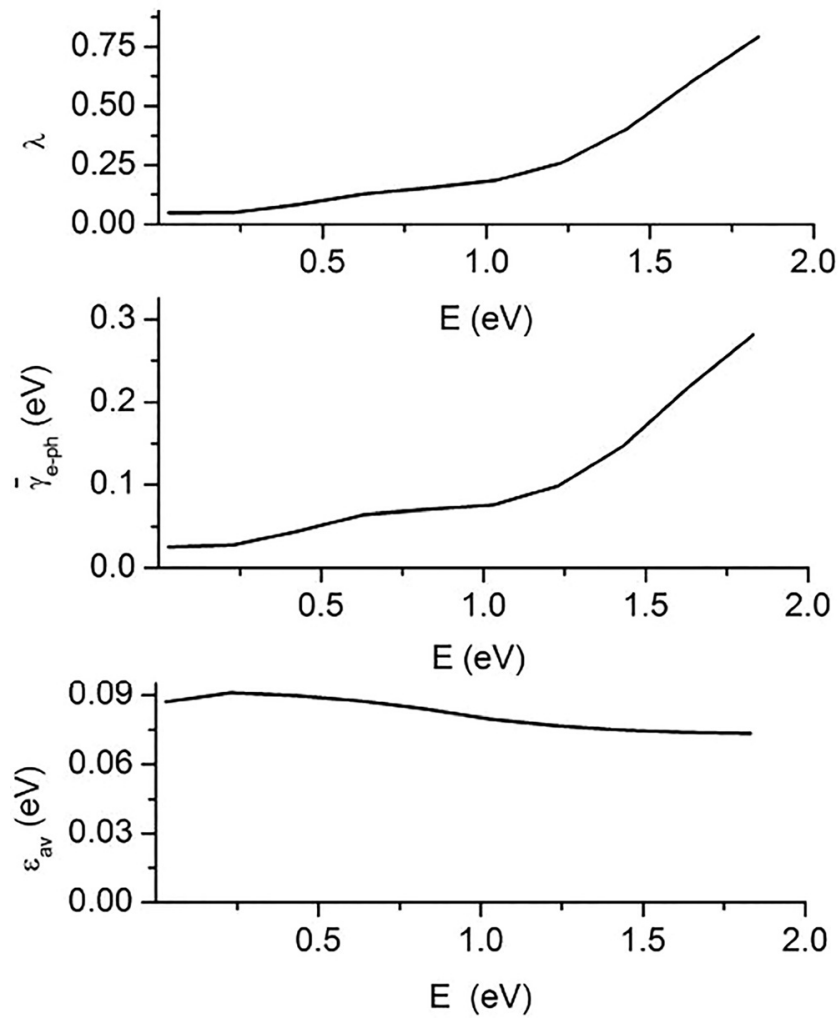

FIG. 4. Energy dependence of the constant of electron-phonon scattering $\lambda$, the rate of the scattering $\gamma_{e-p h}$, and the averaged value of the emitted phonon energy $\epsilon_{a v}$.

leads to the reduction of $\tau$. In other words, the reduction of the electron relaxation time is determined by the expansion of the space of electronic states available for the electron that looses its energy via phonon emission. Note that the changes of $P_{a v}(E)$ in Fig. 5 are very similar to that observed in our previous study of the electron dynamics. ${ }^{26}$
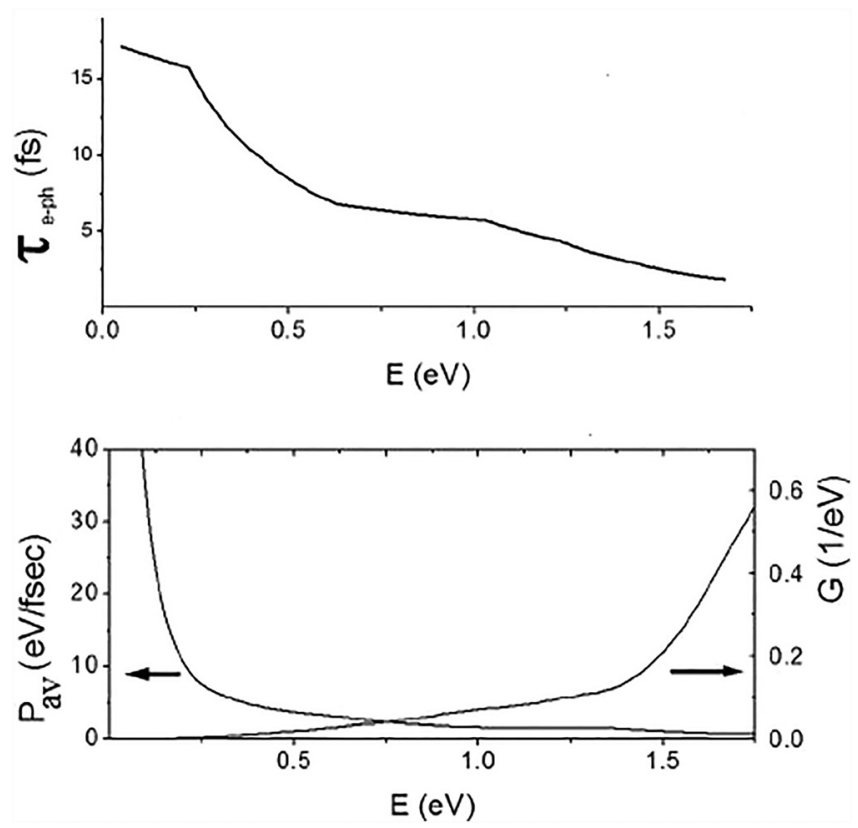

FIG. 5. Energy dependencies of the electron relaxation time $\tau_{e-p h}(E)$, averaged rate of the single phonon emission $P_{a v}(E)$ and density of the electron states $G(E)$.
The available literature data on the electron relaxation time in $G a N$ are contradictory. In Refs. 42 and 43, the electron-phonon relaxation time has been evaluated by using the Frohlich theory of electron-phonon scattering which employs the experimental values of dielectric constants $\varepsilon(\infty), \varepsilon(0)$ and phonon $\omega_{L O}, \omega_{T O}$ frequencies. This estimation of relaxation time, $10 \mathrm{fs}$, corresponds well to our energy-averaged data. However, the energy dependence of the relaxation time has not been investigated.

The energy dependence of a relaxation time was investigated in Refs. 38 and 44. Bulutay et al., ${ }^{38}$ relying on the modified Frohlich's electron-phonon matrix elements, performed the calculations of phonon-assisted scattering rate in GaN taking into account the coupling of excited electrons with long-wave $L O$ and TO phonon modes. Yamakawa et al $^{44}$ extended the model of Ref. 38 by adding the calculation of deformation potentials in semi-phenomenological rigid-ion model. According to the calculations in Refs. 38 and 44, the relaxation rate increases with the excess energy, although this growth is not as rapid as in our first-principle calculations. They found also that the TO-like scattering rate is more than two orders of magnitude lower than that of the LO-like scattering in contrast with our Fig. 3.

Let us note in this connection that in recent $a b$ initio studies of electron-phonon scattering in polar crystals (Sjakste $e t$ al. $^{45}$ and Verdi and Guistino ${ }^{46}$ ), it was shown that the phenomenological Frohlich's expression properly reproduces the long-range scattering probability only for LO phonons with small wave vectors, covering the central part of the Brillouin zone. In the rest part of Brillouin zone, the electron interactions with LO-like phonons are of the short-range nature.

The $a b$ initio probabilities of the electron scattering at TO-like and short-wavelength TA-like and LA-like phonons are comparatively low because of their short range nature. But as the quantity of the electron-phonon processes with the short-range interaction in the high-excitation regime predominates so their contribution to the relaxation rate turns out to be comparable with the contribution from the longwavelength LO phonons as it is shown in Fig. 3.

$A b$ initio calculations in $\mathrm{GaAs}^{47}$ also show that Frohlich's expression strongly overestimates the contribution of LO phonon to the hot electrons' relaxation and underestimates a contribution of TO branches.

Dasgupta et $a{ }^{48}{ }^{48}$ in their experimental work came to the conclusion that the relaxation rate decreases with excess energy. However, their estimation of the relaxation rate and time was not direct since they were based on the measurements of the inelastic mean free path (IMFP) of electrons in a hot electron transistor with $G a N$ as the base layer. The increase of the IMFP with the excess energy can be associated with both the increase of the electron velocity and the reduction of the electron-defect scattering. The discrepancy between our ab initio calculated data and experimental data of Ref. 48 deserves a special study.

In Fig. 6, the results of our calculations for three distribution curves, $\bar{S}(E), f_{0}(E)$, and $f_{1}(E)$, are shown. Our aim is to compare the values of $f_{0}(E)$ and $f_{1}(E)$, so we can omit the factor $S_{0}(t) / \epsilon_{0}$. Two parameters in our evaluations are 

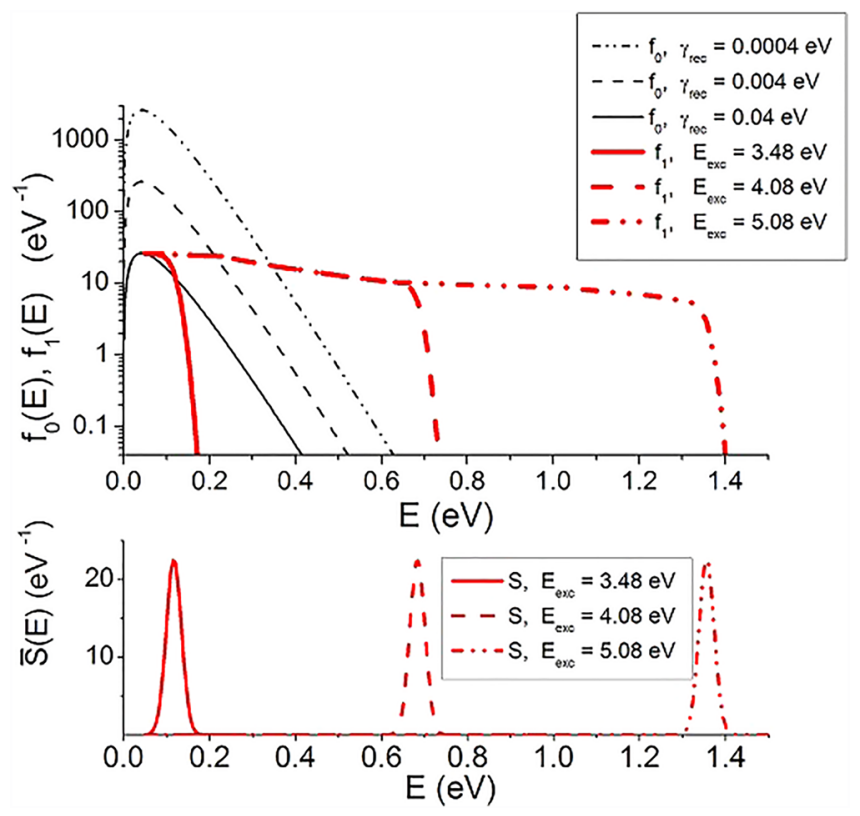

FIG. 6. The energy dependencies of the $f_{0}, f_{1}, \bar{S}$ distribution functions.

determined by the experimental conditions, namely, the excitation energy $E_{\text {exc }}$ and the rate of electron-hole recombination and trapping $\gamma_{\text {rec }}$. We analyse the variation of the distribution functions with respect to $E_{\text {exc }}$ by calculating the $\bar{S}(E)$ and $f_{1}(E)$ functions for three values of $E_{\text {exc }}: 3.48,4.08$, and $5.08 \mathrm{eV}$, which correspond to the lower edge, the center, and the top of our energy interval of interest. The value of $\gamma_{\text {rec }}$ is a "technological" parameter that strongly depends on the concentration of impurities and intrinsic defects in a crystal. We study its influence on the distribution functions by varying it from $0.04 \mathrm{eV}$, the value typical for the rate of electron-phonon scattering, up to the values of 10 and 100 times less, is going to the pico-second times of recombination and trapping processes.

We see that the function $\bar{S}(E)$ has a simple Gaussian shape with a peak energy increasing almost linearly with a rise of $E, E_{\text {exc }}$. By increasing $E_{\text {exc }}$ the upper boundary of the distribution $f_{1}(E)$ shifts to a higher energy, but the values of the $f_{1}(E)$ function do not change markedly, keeping at the level about $40 \mathrm{eV}^{-1}$. The functions $\bar{S}(E)$ and $f_{1}(E)$ of $G a N$ appear to be similar to those calculated before for $\mathrm{ZnO}{ }^{26}$ despite a different method of calculating, based on the linear muffin-tin orbital approach. We also see that when $\gamma_{\text {rec }}$ is taken to be close to the rate of electron-phonon scattering $\gamma_{e-p h}$, then the values of $f_{1}(E)$ and $f_{0}(E)$ appear to be comparable. When $\gamma_{\text {rec }}$ is 10 times smaller than $\gamma_{e-p h}(E)$, then $f_{1}(E)$ is also smaller than $f_{0}(E)$, but still not negligible. When $\gamma_{\text {rec }}$ is of two orders lower than $\gamma_{e-p h}(E)$, then the value of $f_{1}(E)$ becomes relatively small, it does not exceed $1.5 \%$ of $f_{0}(E)$.

\section{CONCLUSIONS}

On the basis of the kinetic theory, we derived the equation for the quasi-equilibrium distribution function of electrons in semiconductors excited by strong long-lasting external irradiation. The leading role in the formation of the carriers' distribution is associated with the inelastic scattering of electrons by phonons. The solution essentially describes a stable flow of electrons downward in an energy scale. In the case of wide-bandgap material, we can allocate two parts. One of them $f_{0}(E)$ describes the accumulation of electrons above the bottom of the conduction band and has a conventional Fermi-like shape with a width of the order of phonon spectrum. Its size depends both on the level of excitation and on the rate of "leakage" of electrons through the process of electron-hole recombination and electron trapping by the defects. The effective temperature and chemical potential generally depend on an occupation number of the excited optical phonon.

When the rate of recombination of electrons and holes and capture electrons on defects is much smaller than the characteristic time of electron-phonon relaxation then a kind of "bottleneck" arises in that flow. The high-energy "tail" $f_{1}(E)$ appears as a result of a balance between the supply from an external source and leakage. This second contribution is much smaller in size but extends much further over the conduction band. The form of the "tail" depends on the band structure and on the rate of electron-phonon energy relaxation. Interestingly, its shape and scale do not depend on the rate of recombination and trapping. The depth of its penetration in the conduction band depends on the rate of excitation of electrons from the valence band to the conduction one and can be comparable with the magnitude of the band gap $E_{g}$ above the bottom of the conduction band.

Based on the theory, we have carried out the firstprinciple calculations for the characteristics of electronphonon scattering and the distribution functions for $G a N$ in the wurtzite structure. A good quality of the calculated electron and phonon band structures is confirmed by the comparison with the available experimental and theoretical data. By calculating the spectral distribution of the electron-phonon scattering (Eliashberg function), we have revealed that the contribution of the LO-phonon branch to the electron relaxation rate is comparable with that of the TO-phonon branch; the contribution of the acoustic branch, although markedly smaller, is not negligible. We also find that the electronphonon relaxation time decreases with the rise of the electron excess energy, which is determined by the increase in the electron density of states.

Calculating the shape of electron distributions we find that when the rate of electron-phonon scattering is comparable with the rate of recombination and electron trapping by lattice defects then the values of the low-energy component are comparable with those of the high-energy "tail" of distribution. However, when the rate of recombination decreases then the value of the low-energy term becomes bigger. When the rate of electron recombination/trapping is two orders smaller, i.e., these processes occur in the picosecond energy range, the tail distribution does not exceed $1.5 \%$ of the lowenergy distribution.

Considering $G a N$ as a typical example, we conclude that the high-energy correction has to be taken into account in the study of the behavior of highly excited charge carrier in a wide-bandgap semiconductor compounds owing a high rate of electron-phonon scattering. 
The two-photon photoemission experiments ${ }^{28,49-52}$ are able to measure the electron distributions in energy and momentum space. Hence, we expect that quasi-equilibrium "tails" can manifest themselves in $2 P P E$ measurements.

The length of the high-energy "tail" may cover a substantial part of the conduction band. Its contribution to the total concentration of non-equilibrium carriers may be comparable to that of the conventional Fermi part and thus can have a significant influence on the transport properties of the irradiated crystal. The presence of the "tail" should lead to a contribution in the conductivity from the carriers which transfer the charges through highly excited states. One can expect that this contribution should have some specific temperature dependence and should only weakly depend of doping.

The experimental evidence of high-energy "tail" already have been observed in experiments on $\mathrm{NaCl}, \mathrm{KCl}, \mathrm{KBr}$ excited by a powerful electron beam. ${ }^{53}$ The high-energy mobility was measured and a quasi-metallic behavior of high-energy conductivity was established.

\section{ACKNOWLEDGMENTS}

The work was supported by RFBR, research Project No. 15-02-00293 and the Tomsk State University Competitiveness Improvement Program. V.G.T. acknowledges the support of Ministry of Education and Science of Russian Federation base part 101. The calculations have been performed by using the URAN cluster of the Institute of mathematics and mechanics in the Urals branch of the Russian Academy of Sciences and SKIF CYBERIA cluster of Tomsk State University.

${ }^{1}$ A. Othonos, J. Appl. Phys. 83, 1789 (1998)

${ }^{2}$ J. Shah, Ultrafast Spectroscopy of Semiconductors and Semiconductor Nanostructures (Springer, New York, 1999).

${ }^{3}$ E. Bringuier, Phys. Rev. B 57, 2280 (1998).

${ }^{4}$ W. Tisdale, K. Williams, B. Timp, D. Norris, E. Aydil, and X. Y. Zhu, Science 328, 1543 (2010).

${ }^{5}$ M. Dur, S. Goodnick, S. Pennathur, J. Wager, M. Reigrotzki, and R. Redmer, J. Appl. Phys. 83, 3176 (1998).

${ }^{6}$ Y. Sun, S. Boggs, and R. Ramprasad, Appl. Phys. Lett. 101, 132906 (2012).

${ }^{7}$ F. Bertazzi, M. Moresco, and E. Bellotti, J. Appl. Phys. 106, 063718 (2009).

${ }^{8}$ C. Wang, L. Jiang, F. Wang, X. Li, Y. Yuan, and H. Tsai, Phys. Lett. A 375, 3200 (2011).

${ }^{9}$ A. A. Grinberg and S. Luryi, Phys. Rev. Lett. 65, 1251 (1990).

${ }^{10}$ Y. Gurevich, G. Logvinov, Y. Drogobitskiy, A. Carballo-Sanchez, and J. Salazar, J. Appl. Phys. 91, 183 (2002).

${ }^{11}$ D. von der Linde, K. Sokolowski-Tinten, and J. Bialkowski, Appl. Surf. Sci. 109-110, 1 (1997).

${ }^{12}$ V. P. Zhukov, V. G. Tyuterev, E. V. Chulkov, and P. M. Echenique, Int. J. Photoenergy 2014, 738921.

${ }^{13}$ M. Neges, K. Schwarzburg, and F. Willig, Sol. Energy Mater. Sol. Cells 90, 2107 (2006).

${ }^{14}$ E. Tea, H. Hamzeh, and F. Aniel, J. Appl. Phys. 110, 113108 (2011).

${ }^{15}$ M. Harb, R. Ernstorfer, T. Dartigalongue, C. Hebeisen, R. Jordan, and R. Miller, J. Phys. Chem. B 110, 25308 (2006).

${ }^{16}$ J. Callan, A.-T. Kim, L. Huang, and E. Mazur, Chem. Phys. 251, 167 (2000).

${ }^{17}$ G. Fasol, W. Hackenberg, H. P. Hughes, K. Ploog, E. Bauser, and H. Kano, Phys. Rev. B 41, 1461 (1990).
${ }^{18}$ P. Supancic, U. Hohenester, P. Kocevar, D. Snoke, R. Hannak, and W. Ruhle, Phys. Rev. 53, 7785 (1996).

${ }^{19}$ M. Asche and O. G. Sarbei, Phys. Status Solidi B 141, 487-491 (1987).

${ }^{20}$ R. F. Leheny, J. Shah, R. L. Fork, C. V. Shank, and A. Migus, Solid State Commun. 31, 809-813 (1979).

${ }^{21}$ C. Collier, B. Born, X. Jin, and J. Holzman, Appl. Phys. Lett. 103, 072106 (2013).

${ }^{22}$ C. M. Collier, B. Born, X. Jin, and J. F. Holzman, Proc. SPIE 8845, $88450 \mathrm{U}(2013)$.

${ }^{23}$ U. Hohenester, P. Supancic, P. Kocevar, X. Q. Zhou, W. Kutt, and H. Kurz, Phys. Rev. B 47, 13233 (1993).

${ }^{24}$ S. S. Prabhu, A. S. Vengurlekar, S. K. Roy, and J. Shah, Phys. Rev. B 51, 14233 (1995).

${ }^{25}$ V. Zhukov and V. Tyuterev, Phys. Solid State 54, 2173 (2012).

${ }^{26}$ V. Zhukov, V. Tyuterev, and E. Chulkov, J. Phys.: Condens. Matter 24, 405802 (2012).

${ }^{27}$ V. G. Tyuterev, V. P. Zhukov, P. M. Echenique, and E. V. Chulkov, J. Phys.: Condens. Matter 27, 025801 (2015).

${ }^{28}$ H. Tanimura, J. Kanasaki, K. Tanimura, J. Sjakste, N. Vast, M. Calandra, and F. Mauri, Phys. Rev. B 93, 161203(R) (2016).

${ }^{29}$ G. M. Eliashberg, Sov. Phys. JETP 11, 696 (1960).

${ }^{30}$ A. H. Nayfeh, "General weakly nonlinear systems," in Introduction to Perturbation Techniques (Wiley-Interscience, NY, 1981), p. 177.

${ }^{31} \mathrm{R}$. Loudon, The Quantum Theory of Light (Oxford University Press Inc., New York, 1983).

${ }^{32}$ S. Baroni, S. de Gironcoli, and A. Dal Corso, Rev. Mod. Phys. 73, 515 (2001).

${ }^{33}$ G. B. Bachelet, D. R. Hamann, and M. Schlüter, Phys. Rev. B 26, 4199 (1982).

${ }^{34}$ J. P. Perdew and A. Zunger, Phys. Rev. B 23, 5048 (1981).

${ }^{35}$ A. Dal Corso, S. Baroni, R. Resta, and S. de Gironcoli, Phys. Rev. B 47, 3588 (1993).

${ }^{36}$ A. Benassi, PW SCF epsilon.x users manual, Technical Report, Physics Department, Universita degli Studi di Modena e Reggio Emilia, Report No. INFM/S3, 2008

${ }^{37}$ B. G. Streetman and S. Banerjee, Solid State Electronic Devices, 5 th ed. (Prentice Hall, New Jersey, 2000), p. 524.

${ }^{38}$ C. Bulutay, B. K. Ridley, and N. A. Zakhleniuk, Phys. Rev. B 62, 15754 (2000).

${ }^{39}$ S. Syed, J. B. Heroux, Y. J. Wang, M. J. Manfra, R. J. Molnar, and H. L. Stormer, Appl. Phys. Lett. 83, 4553 (2003).

${ }^{40}$ T. Ruf, J. Serrano, M. Cardona, P. Pavone, M. Pabst, M. Krisch, M. D. Astuto, T. Suski, I. Grzegory, and M. Leszczynski, Phys. Rev. Lett. 86, 906 (2001).

${ }^{41}$ B. C. Lee, K. W. Kim, M. Dutta, and M. A. Stroscio, Phys. Rev. B 56, 997 (1997).

${ }^{42}$ N. Stanton, A. Kent, A. Akimov, P. Hawker, T. Cheng, and C. T. Foxon, J. Appl. Phys. 89, 973 (2001).

${ }^{43}$ S. J. Sheih, K. Tsen, D. Ferry, A. Botchkarev, B. Sverdlov, A. Salvador, and H. Morkoc, Appl. Phys. Lett. 67, 1757 (1995).

${ }^{44}$ S. Yamakawa, S. Aboud, M. Saranit, and S. M. Goodnick, Semicond. Sci. Technol. 19, S475 (2004).

${ }^{45}$ J. Sjakste, N. Vast, M. Calandra, and F. Mauri, Phys. Rev. B 92, 054307 (2015).

${ }^{46}$ C. Verdi and F. Giustino, Phys. Rev. Lett. 115, 176401 (2015).

${ }^{47}$ M. Bernardi, D. Vigil-Fowler, C. S. Ong, J. B. Neaton, and S. G. Louie, PNAS 112, 5291 (2015)

${ }^{48}$ S. S. Dasgupta, J. L. Nidhi, A. Raman, C. Hurni, G. Gupta, J. Speck, and U. Mishra, Appl. Phys. Express 6, 034002 (2013).

${ }^{49}$ W. A. Tisdale, M. Muntwiler, D. J. Norris, E. S. Aydil, and X.-Y. Zhu, J. Phys. Chem. C 112, 14682 (2008).

${ }^{50}$ J.-C. Deinert, D. Wegkamp, M. Meyer, C. Richter, M. Wolf, and J. Stahler, Phys. Rev. Lett. 113, 057602 (2014).

${ }^{51}$ K. Onda, B. Li, and H. Petek, Phys. Rev B 70, 045415 (2004).

${ }^{52}$ A. Argondizzo, S. Tan, and H. Petek, J. Phys. Chem. C 120, 12959 (2016).

${ }^{53} \mathrm{D}$. Vaisburd, in Proceedings of the II International Conference on Space Charge in Solid Dielectrics. Antibes-Juan-les-Pins, Supplement a la Revie Le Vide I Science, Technique et Applications $N$ 275, JanvierFevrier-Mars (1995), pp. 126-136. 